\title{
Wheelchair ergonomic hand drive mechanism use improves wrist mechanics associated with carpal tunnel syndrome
}

\author{
Lisa A. Zukowski, MA; ${ }^{*}$ Jaimie A. Roper, MS; ${ }^{1}$ Orit Shechtman, PhD, OTR/L; ${ }^{2}$ Dana M. Otzel, PhD; ${ }^{1,3}$ Patty \\ W. Hovis, MSESS; ${ }^{1}$ Mark D. Tillman, PhD ${ }^{1,4}$ \\ Departments of ${ }^{1}$ Applied Physiology and Kinesiology and ${ }^{2}$ Occupational Therapy, University of Florida, Gainesville, \\ FL; ${ }^{3}$ Geriatric Research, Education, and Clinical Center, North Florida/South Georgia Veterans Health System, \\ Gainesville, FL; ${ }^{4}$ Department of Kinesiology and Health Promotion, Troy University, Troy, AL
}

\begin{abstract}
Among conventional manual wheelchair (CMW) users, $49 \%$ to $63 \%$ experience carpal tunnel syndrome (CTS) that is likely induced by large forces transmitted through the wrist and extreme wrist orientations. The ergonomic hand drive mechanism (EHDM) tested in this study has been shown to utilize a more neutral wrist orientation. This study evaluates the use of an EHDM in terms of wrist orientations that may predispose individuals to CTS. Eleven adult full-time CMW users with spinal cord injury participated. Motion data were captured as participants propelled across a flat surface, completing five trials in a CMW and five trials in the same CMW fitted with the EHDM. Average angular wrist orientations were compared between the two propulsion styles. Use of the EHDM resulted in reduced wrist extension and ulnar deviation. The shift to more neutral wrist orientations observed with EHDM use may reduce median nerve compression.
\end{abstract}

Key words: assistive technology, biomechanics, kinematics, median nerve, mobility, propulsion, quality of life, spinal cord injury, upper limb, wheelchair.

\section{INTRODUCTION}

Carpal tunnel syndrome (CTS) is an overuse injury that develops in 49 to 63 percent of conventional manual wheelchair (CMW) users [1-4]. CTS is caused by compression of the median nerve in the carpal tunnel and is characterized by pain and numbness in the hand and wrist that progressively worsens if left untreated [5-6]. Because
CMW users have an increased reliance on their upper limbs for mobility and weight relief in addition to performing activities that require grasping and reaching, pain in the hand and wrist can be particularly debilitating. Pain associated with CTS may prevent individuals from maintaining their independence and from participating in activities of daily living, lessening the quality of life of these individuals [7]. Previous research has noted that future work should focus on new propulsion techniques that reduce stress on the wrist with each push in order to lessen the risk of developing CTS [8]. Reducing stress on the wrist is especially important because CTS is an overuse injury and median nerve damage typical of CTS is cumulative [9]. Further, a typical CMW user pushes on the pushrims 2,500 times each day with just 40 min of propulsion, which is probably an underestimation [10-11].

CTS is likely induced by a combination of (1) large forces transmitted through the wrist and (2) an extreme range of wrist motion, both of which can compress the

Abbreviations: $\mathrm{CMW}=$ conventional manual wheelchair, $\mathrm{CTS}=$ carpal tunnel syndrome, $\mathrm{EHDM}=$ ergonomic hand drive mechanism.

*Address all correspondence to Lisa A. Zukowski, PhD; University of North Carolina at Chapel Hill, Division of Physical Therapy, 3070 Bondurant Hall, CB\# 7135, Chapel Hill, NC 27599-7135; 919-966-9797; fax: 919-966-3678.

Email: lazuk@email.unc.edu

http://dx.doi.org/10.1682/JRRD.2013.09.0211 
median nerve within the carpal tunnel and are typical of CMW propulsion. For any given muscular force, the joint reaction force and therefore median nerve compression increases as the deviation from neutral orientation increases [12]. Therefore, compression of the nerve is greater with applied forces at extreme ranges of motion than compression of the nerve during periods of force application with more neutral wrist positioning. Keir et al. observed that wrist orientation beyond $48.6^{\circ}$ of flexion, $32.7^{\circ}$ of extension, $21.8^{\circ}$ of radial deviation, or $14.5^{\circ}$ of ulnar deviation leads to carpal tunnel pressure greater than the critical threshold of $30 \mathrm{~mm} \mathrm{Hg}$ [13]. Therefore, these wrist orientations can be defined as extreme or outside the limits of the neutral range of orientation. When this critical pressure threshold is exceeded for short periods of time, the temporary effects are consistent with initial stages of CTS [14]. Chronic application in excess of this critical pressure threshold is known to result in nerve damage [15]. Contrary to the findings of the previously mentioned studies, Boninger et al. observed a relationship between an increased wrist flexion and extension range of motion and improved median nerve conduction during CMW propulsion [16]. However, this increased range of motion was also related to lower cadence and lower forces. Boninger et al. attribute the improved median nerve health to the longer, smoother propulsion style characterized by decreased cadence and peak force and only cite the increased range of motion as a lesser, corollary factor [16]. Therefore, a propulsion style that utilizes extreme wrist orientations as specified by Keir et al. [13] may still pose a risk for developing CTS as suggested by Veeger et al. [17].

While various researchers have examined the metabolic cost and mechanical efficiency of previous leverpropelled wheelchair designs, the analysis of wrist range of motion during lever propulsion has either been neglected or assumed to be beneficial [18-21]. The ergonomic hand drive mechanism (EHDM) tested in this study considers user anthropometrics that other leverpropelled designs ignore and has previously been shown to utilize a more neutral range of wrist orientation [2223]. Ideally, the EHDM would utilize a wrist orientation within the limits as specified by Keir et al. to reduce carpal tunnel pressure and therefore the risk of developing CTS [13]. The purpose of this project was to evaluate the use of the EHDM in terms of wrist orientations that may predispose individuals to CTS.

\section{METHODS}

\section{Participants}

A heterogeneous population of 11 adult full-time CMW users, including both persons with paraplegia and tetraplegia, was recruited for participation (Table 1). All participants were determined to be medically and functionally stable and at least 6 mo postinjury before inclusion. The protocol was approved by the University of Florida Institutional Review Board and all participants signed an informed consent before testing started.

Table 1.

Participant demographics.

\begin{tabular}{|c|c|c|c|c|c|}
\hline Subject & Sex & Age (yr) & Body Mass (kg) & Height (m) & Lesion Level \\
\hline \multicolumn{6}{|c|}{ Persons with Paraplegia } \\
\hline 1 & M & 45 & 81.8 & 1.85 & $\mathrm{~T} 4$ \\
\hline 2 & M & 23 & 68.2 & 1.73 & T6 \\
\hline 3 & M & 53 & 70.5 & 1.78 & $\mathrm{~T} 7$ \\
\hline 4 & $\mathrm{~F}$ & 19 & 59.1 & 1.63 & $\mathrm{~T} 8$ \\
\hline 5 & M & 31 & 59.5 & 1.73 & T9 \\
\hline \multicolumn{6}{|c|}{ Persons with Tetraplegia } \\
\hline 8 & M & 25 & 84.1 & 1.85 & C6 \\
\hline 9 & M & 49 & 95.5 & 1.91 & C6-C7 \\
\hline 10 & $\mathrm{~F}$ & 46 & 59.1 & 1.70 & $\mathrm{C} 7-\mathrm{T} 1$ \\
\hline
\end{tabular}




\section{Equipment}

The EHDM utilizes a cam pawl and ratchet mechanism that grabs onto the tire during forward propulsion and releases it during the recovery phase (Figure 1). The EHDM was attached to the axle of both wheels on a CMW (QuickieGP, Sunrise Medical LLC; Fresno, California) and could be rotated to the back of the wheelchair when not in use, as previously described in Zukowski et al. [23]. With this setup, the same wheelchair was used for all testing and maintained all the same settings for each propulsion style condition. Lever length and handgrip orientation could be modified and were adjusted according to user anthropometrics and preference (Figure 2). Thirty-five reflective markers were placed on the participants in accordance with the Vicon upper limb and plug-in-gait models, excluding all markers distal to the knee. Motion data were captured by 11 Vicon MX series cameras (Vicon; Oxford, United Kingdom) at a sampling rate of $120 \mathrm{~Hz}$.

\section{Protocol}

Prior to testing, participants transferred into the CMW fitted with the EHDM and propelled themselves until they were comfortable with both CMW and EHDM

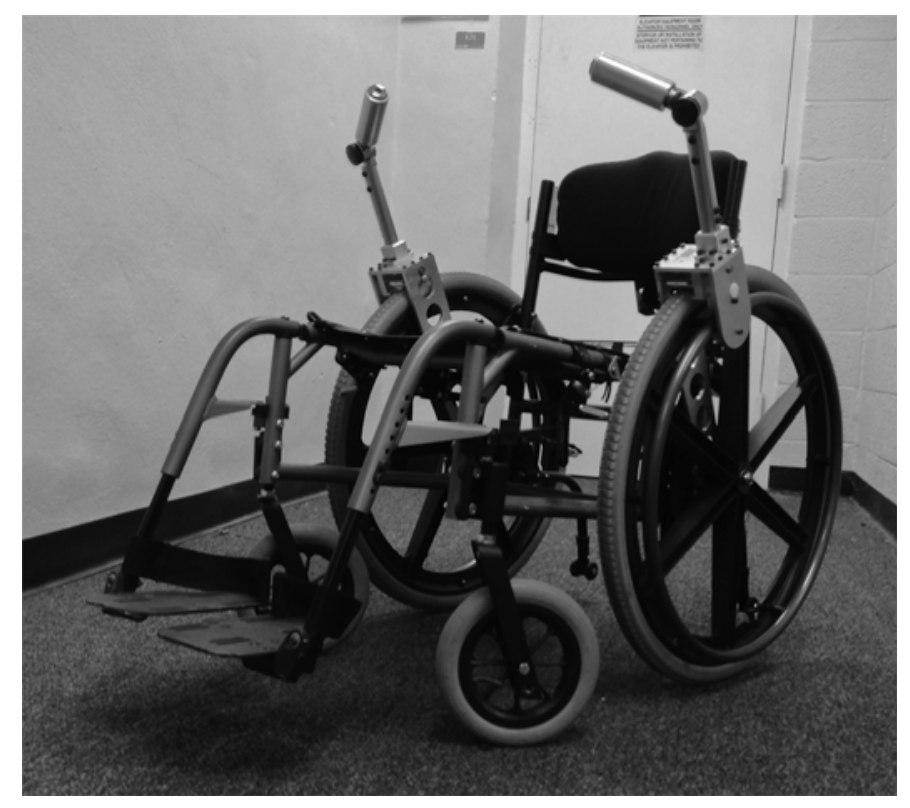

\section{Figure 1.}

Ergonomic hand drive mechanism attached to axle of both wheels on conventional manual wheelchair. Reprinted with permission from Zukowski et al. [23].

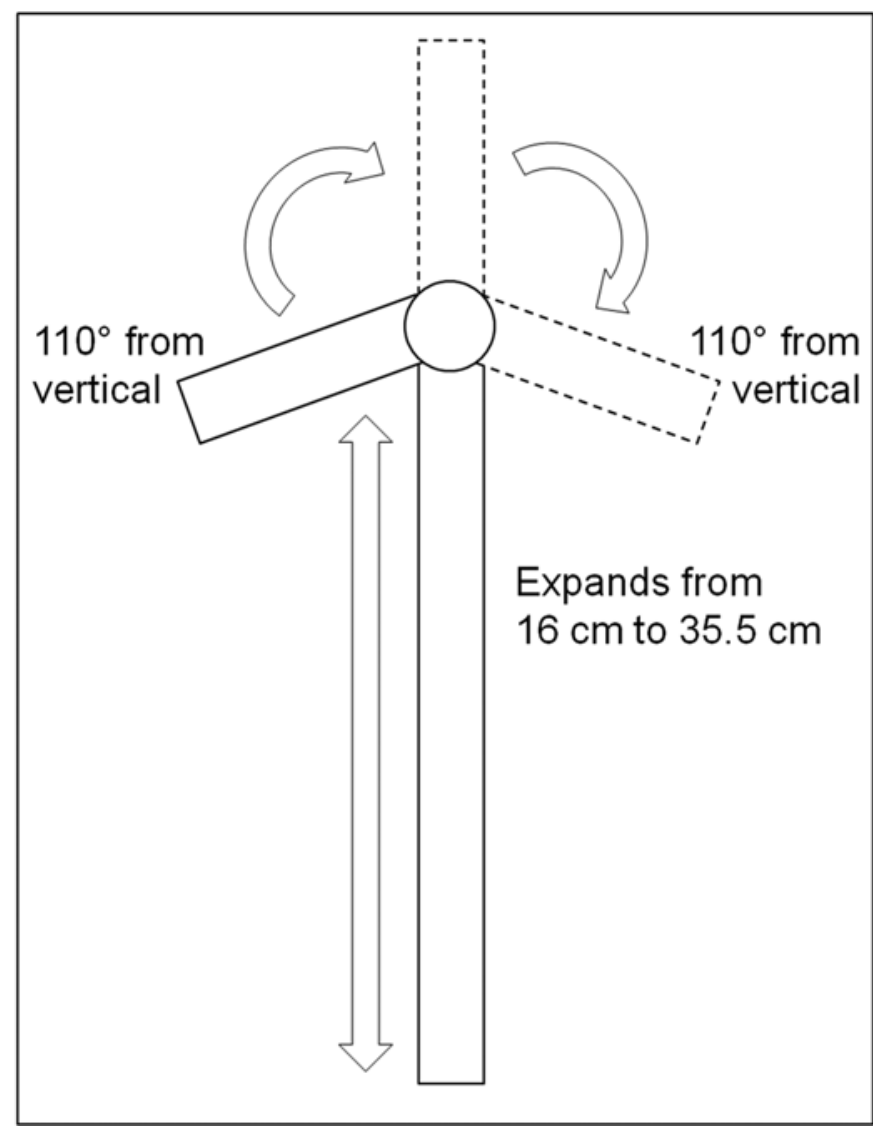

Figure 2.

Schematic of adjustable lever length and pivoting handgrip of ergonomic hand drive mechanism. Reprinted with permission from Zukowski et al. [23].

propulsion. During testing, participants propelled at a self-selected speed across a length of $8 \mathrm{~m}$, completing five trials in a CMW and five trials in the same CMW fitted with the EHDM. The two sets of trials were randomized to prevent a fatigue bias. Participants were told to propel themselves at a comfortable speed in order to prevent artificial constraints on propulsion. Angular kinematics of the wrist in the planes of flexion and extension and radial and ulnar deviation were computed with Nexus version 1.8.5 (Vicon) and BodyBuilder version 3.6.1 (Vicon) using the XZY Euler angles sequence.

\section{Analysis}

A custom MATLAB program (MathWorks; Natick, Massachusetts) was written to filter data with a fourth-order Butterworth low-pass filter (cutoff frequency of $10 \mathrm{~Hz}$ chosen based on residual analysis). Only trials that 
contained at least one complete push were included in the analysis. A complete push was operationally defined as including both a push phase (from hand contact with the rim to release of the rim) and a recovery phase (from release of the rim to hand contact with the rim). After all data were filtered, each push was separated into a push phase and a recovery phase and then cut down to exclude the recovery phase. At least one push phase was analyzed per trial, but all push phases recorded were analyzed. Each push phase, heretofore referred to as a push, was then time normalized by percentage to 100 percent. All normalized pushes for each trial were combined to calculate an average push per trial and then these trial averages were subsequently combined to calculate an average push per participant. This average push was then divided into 10 consecutive percentile intervals, each constituting 10 percent of the total push. Average angular orientation of the right wrist in the planes of flexion and extension and radial and ulnar deviation were determined during each percentile interval. These angular values were then compared between the two propulsion style conditions using paired samples $t$-tests $(\alpha=0.01$, adjusted for multiple comparisons). Wrist orientations that exceed critical pressure thresholds in the carpal tunnel as specified by Keir et al. (beyond $48.6^{\circ}$ of flexion, $32.7^{\circ}$ of extension, $21.8^{\circ}$ of radial deviation, or $14.5^{\circ}$ of ulnar deviation) have been operationally defined as extreme or exceeding the limits of the neutral range of wrist orientations [13]. Statistical analyses were completed using SPSS version 17.0 (IBM Corporation; Armonk, New York).

\section{RESULTS}

\section{Velocity and Cadence}

Although no hypotheses were made concerning velocity and cadence, these variables were analyzed between the two conditions to facilitate interpretation of the proposed analyses. Participants selected a speed of $0.67 \pm 0.18 \mathrm{~m} / \mathrm{s}$ (mean \pm standard deviation) using the EHDM versus $1.03 \pm 0.22 \mathrm{~m} / \mathrm{s}$ using the CMW $(p<$ 0.001 ) and a cadence of $47.8 \pm 7.1$ pushes per minute using the EHDM versus $56.0 \pm 10.0$ pushes per minute using the CMW $(p=0.009)$.

\section{Wrist Flexion and Extension}

Wrist orientation exceeded the limits of the neutral range of wrist extension but not wrist flexion while using both the EHDM and the CMW (Figures 3-4). Specifi-

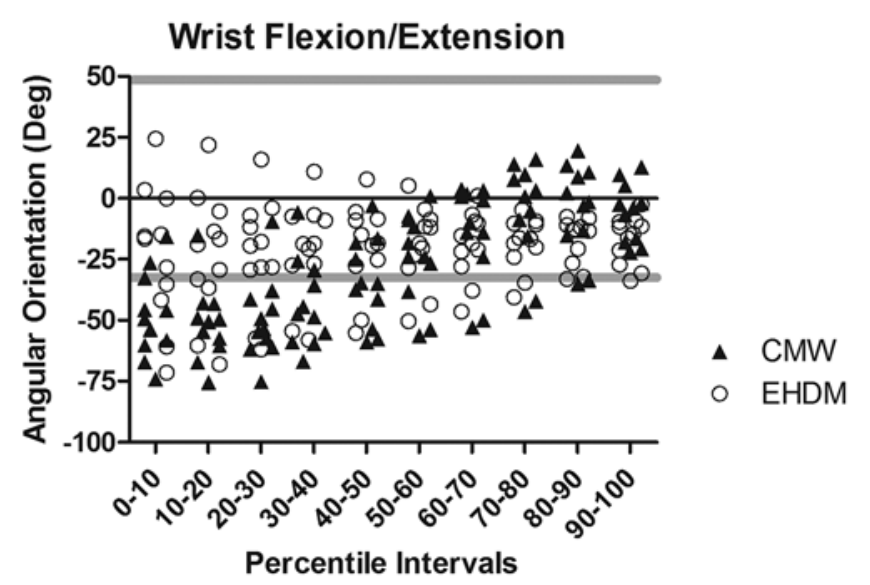

Figure 3.

Wrist flexion and extension (positive and negative values) of both propulsion styles. Horizontal gray lines represent limits of neutral range of wrist orientations. $\mathrm{CMW}=$ conventional manual wheelchair, EHDM = ergonomic hand drive mechanism.

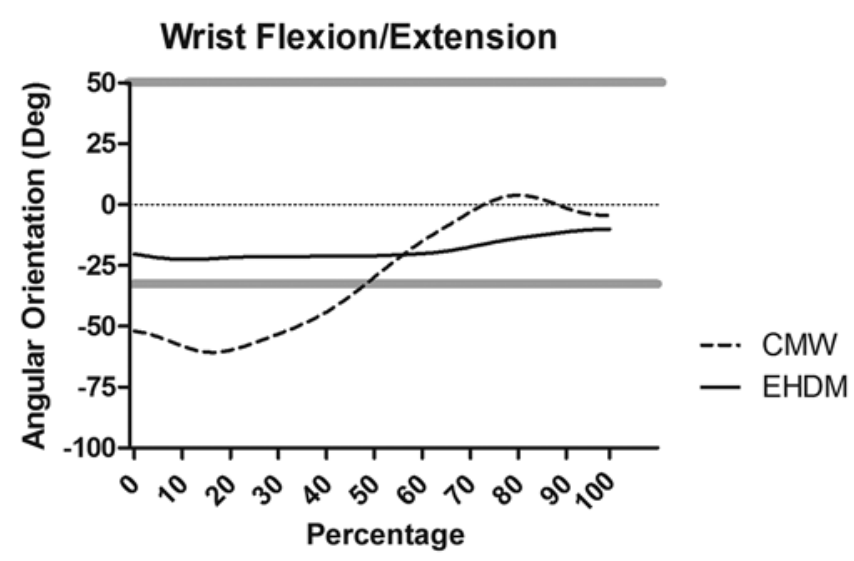

Figure 4.

Typical normalized time series in direction of flexion and extension for both propulsion styles. Third trial for each propulsion condition from single participant is depicted. Horizontal gray lines represent limits of neutral range of wrist orientations. $\mathrm{CMW}=$ conventional manual wheelchair, EHDM = ergonomic hand drive mechanism.

cally, during percentiles 20 to 40 of the push, use of the EHDM resulted in an average of $24.5^{\circ}$ less wrist extension than the CMW (Table 2). Further, CMW use resulted in wrist orientation consistently outside of the neutral range of wrist extension during this same 20 percent of the push (Table 2). During percentiles 0 to 20 and 
Table 2.

Paired samples t-tests comparing average flexion and extension of wrist at each percentile interval (negative values represent extension).

\begin{tabular}{lcccc}
\hline Interval (\%) & $\begin{array}{c}\text { Conventional Manual } \\
\left.\text { Wheelchair } \mathbf{(}^{\circ}\right)\end{array}$ & $\begin{array}{c}\text { Ergonomic Hand Drive } \\
\left.\text { Mechanism } \mathbf{(}^{\circ}\right)\end{array}$ & $\boldsymbol{t}$-Test & $\begin{array}{c}\text { Significance } \\
(\boldsymbol{p} \text {-Value) }\end{array}$ \\
\hline $00-10$ & $-48.1 \pm 17.6$ & $-23.3 \pm 28.2$ & 2.272 & 0.05 \\
$10-20$ & $-51.4 \pm 15.6$ & $-23.6 \pm 26.0$ & 2.996 & 0.01 \\
$20-30$ & $-49.7 \pm 17.0$ & $-22.6 \pm 22.6$ & 3.483 & $0.006^{*}$ \\
$30-40$ & $-43.4 \pm 17.9$ & $-21.5 \pm 20.3$ & 3.508 & $0.006^{*}$ \\
$40-50$ & $-34.7 \pm 17.9$ & $-20.5 \pm 18.7$ & 2.915 & 0.02 \\
$50-60$ & $-24.4 \pm 18.6$ & $-19.6 \pm 16.4$ & 1.202 & 0.26 \\
$60-70$ & $-14.2 \pm 20.4$ & $-19.5 \pm 13.9$ & -1.382 & 0.20 \\
$70-80$ & $-6.1 \pm 21.2$ & $-18.7 \pm 10.9$ & -3.264 & $0.009^{*}$ \\
$80-90$ & $-4.3 \pm 18.3$ & $-17.3 \pm 9.3$ & -4.112 & $0.002^{*}$ \\
$90-100$ & $-5.9 \pm 12.2$ & $-17.1 \pm 9.9$ & -4.278 & $0.002^{*}$ \\
\hline
\end{tabular}

Note: Values are mean \pm standard deviation. Shaded cells indicate that mean values exceed limits of neutral range of wrist flexion and/or extension. *Significant result at 0.01 level (two-tailed).

40 to 50 , CMW use resulted in average wrist orientations consistently outside of the neutral range of wrist extension with CMW use although the differences between propulsion style conditions only approached significance (Table 2). During the last 30 percent of the push, EHDM propulsion utilized a wrist orientation within the neutral range of extension, but CMW propulsion exhibited even less wrist extension than EHDM propulsion (Table 2).

\section{Wrist Radial and Ulnar Deviation}

Wrist orientation exceeded the limits of the neutral range of ulnar deviation but never radial deviation when using both the EHDM and the CMW (Figures 5-6). Wrist orientations significantly differed between propulsion style conditions but were within the neutral range of radial and ulnar deviation during the first 20 percent of the push (Table 3). During the last 30 percent of the push, EHDM propulsion resulted in an average of $13.7^{\circ}$ less ulnar deviation than CMW propulsion (Table 3). Further, CMW use resulted in wrist orientation outside of the neutral range of ulnar deviation during this same 30 percent of the push (Table 3). During the 60 to 70 percentile interval, differences between the propulsion style conditions only approached significance, but the same pattern of CMW use exhibiting wrist orientation outside of the neutral range of ulnar deviation was evident (Table 3).

\section{DISCUSSION}

The purpose of this project was to evaluate the use of an EHDM in terms of wrist orientations that may predis-

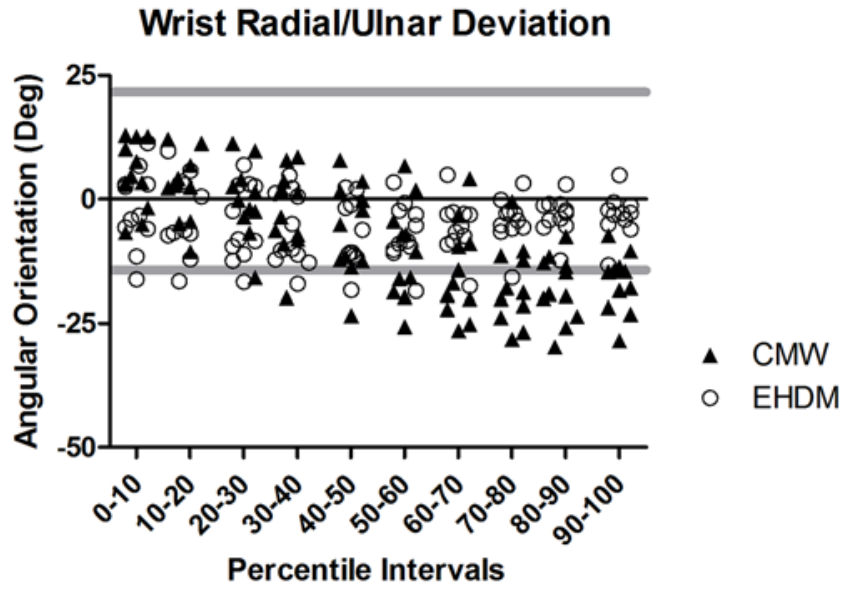

Figure 5.

Wrist radial and ulnar deviation (positive and negative values) of both propulsion styles. Horizontal gray lines represent limits of neutral range of wrist orientations. $\mathrm{CMW}=$ conventional manual wheelchair, EHDM = ergonomic hand drive mechanism.

pose individuals to CTS. In an effort to preserve ecological validity, velocity and cadence were not controlled between the two propulsion conditions, resulting in a faster velocity utilized with the CMW and a lower cadence utilized with the EHDM. The different velocities and cadences utilized may have affected the kinematics recorded, but these data are more representative of preferred propulsion using each wheelchair. Further, Boninger et al. observed wrist orientations at two different speeds $(1.3$ and $2.2 \mathrm{~m} / \mathrm{s})$ and observed that mean maximum flexion, extension, and ulnar deviation angles did 


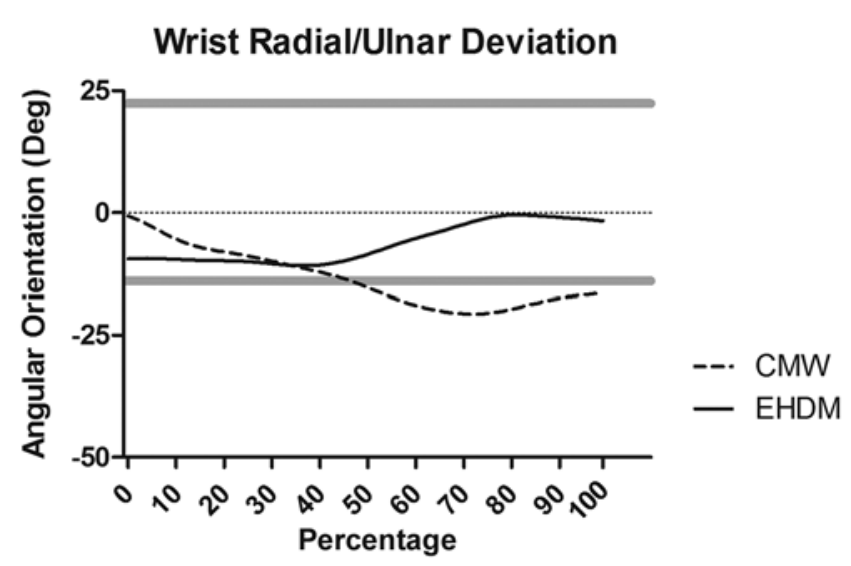

Figure 6.

Typical normalized time series in direction of radial and ulnar deviation for both propulsion styles. Third trial for each propulsion condition from single participant is depicted. Horizontal gray lines represent limit of neutral range of wrist orientations. $\mathrm{CMW}=$ conventional manual wheelchair, $\mathrm{EHDM}=$ ergonomic hand drive mechanism.

not change with speed [10]. The mean maximum radial deviation angle did decrease with increasing speed, but the pattern and timing remained the same [10]. Therefore, the different cadences and velocities utilized should not affect the interpretation of the wrist flexion, extension, and ulnar deviation results.

EHDM use resulted in reduced wrist extension throughout the first half of the push and reduced wrist ulnar deviation throughout the second half of the push compared with CMW use. Perhaps more importantly, EHDM propulsion consistently utilized an average wrist orientation within the limits of the neutral range of wrist positioning in both planes of motion as specified by Keir et al. [13]. CMW propulsion, alternatively, utilizes an average wrist orientation outside of the neutral range of extension during the first half of the push and outside of the neutral range of ulnar deviation during the second half of the push. The size of the carpal tunnel is known to be reduced at extremes of extension and ulnar deviation, which increases carpal tunnel pressure and the likelihood of median nerve compression [17]. Therefore, less time spent in extreme ranges of extension and ulnar deviation as seen with EHDM use may reduce median nerve compression and therefore cumulative median nerve damage typical of CTS.

Two studies observed typical wheelchair users and wheelchair athletes and determined that both groups started the push with wrist extended and radially deviated and moved to ulnar deviation and flexion or extension for the latter half of the push $[10,17]$. The results of the current study exhibit a transition from extreme extension and radial deviation to slight extension and ulnar deviation throughout the push using the CMW, which mirrors previously reported results of CMW propulsion $[10,17]$. Peak moments and forces at the wrist were reported to occur at the beginning and end of the push during the more extreme ranges of motion in both the direction of extension and radial and ulnar deviation $[10,17]$. These large forces and torques compress the median nerve more as deviation from the neutral range of orientation

Table 3.

Paired samples $t$-tests comparing average radial and ulnar deviation of wrist at each percentile interval (negative values represent ulnar deviation).

\begin{tabular}{|c|c|c|c|c|}
\hline Interval (\%) & $\begin{array}{c}\text { Conventional Manual } \\
\text { Wheelchair }\left({ }^{\circ}\right)\end{array}$ & $\begin{array}{c}\text { Ergonomic Hand Drive } \\
\text { Mechanism }\left({ }^{\circ}\right)\end{array}$ & $t$-Test & $\begin{array}{c}\text { Significance } \\
\text { (p-Value) }\end{array}$ \\
\hline$\overline{00-10}$ & $4.9 \pm 7.1$ & $-1.9 \pm 8.1$ & -6.524 & $<0.001^{*}$ \\
\hline $10-20$ & $2.3 \pm 6.9$ & $-3.1 \pm 8.1$ & -3.356 & $0.007^{*}$ \\
\hline $20-30$ & $-0.2 \pm 7.5$ & $-4.9 \pm 7.8$ & -2.122 & 0.06 \\
\hline $30-40$ & $-2.8 \pm 8.5$ & $-6.4 \pm 7.4$ & -1.349 & 0.21 \\
\hline $40-50$ & $-6.2 \pm 9.3$ & $-7.2 \pm 6.7$ & -0.345 & 0.74 \\
\hline $60-70$ & $-14.7 \pm 9.5$ & $-5.6 \pm 5.5$ & 2.930 & 0.02 \\
\hline $70-80$ & $-17.5 \pm 8.2$ & $-4.4 \pm 4.7$ & 4.761 & $0.001^{*}$ \\
\hline $80-90$ & $-18.0 \pm 6.6$ & $-3.4 \pm 3.9$ & 6.849 & $<0.001^{*}$ \\
\hline $90-100$ & $-16.8 \pm 6.0$ & $-3.3 \pm 4.4$ & 7.089 & $<0.001^{*}$ \\
\hline
\end{tabular}

Note: Values are mean \pm standard deviation. Shaded cells indicate that mean values exceed limits of neutral range of wrist radial and/or ulnar deviation. ${ }^{*}$ Significant result at 0.01 level (two-tailed). 
increases [12]. Reducing wrist extension at the beginning of the push and ulnar deviation at the end of the push with EHDM use, therefore, represents an important reduction of peak push forces acting on the wrist and consequently possible compression of the median nerve.

\section{STUDY LIMITATIONS}

This study is limited by a small sample size that includes a heterogeneous group of CMW users. The diversity of the participant group and the greater experience using the CMW versus the EHDM may have increased the variability of the results, which could have reduced the number of significant findings. Future studies should involve a greater number of individuals to allow for separate analyses of different groups of wheelchair users as well as allow for a longer training period with the EHDM.

Additionally, all current study participants used the same wheelchair regardless of the height and weight of the participant. Participants were able to adjust the levers to a preferred length and orientation, but an optimized wheelchair fit could maximize performance. Although not imperative because each individual acted as his or her own control, a better fit to the wheelchair could improve the mechanical efficiency of an individual and alter the utilized wrist range of motion overall. Future studies would use multiple wheelchair sizes to better accommodate a larger range of study participants.

Self-selected speeds and cadences were utilized throughout the study for both testing conditions in order to prevent artificial constraints on propulsion. Not controlling speed and cadence for EHDM and CMW propulsion may have limited the interpretation of the results. The findings of the study performed by Boninger et al. [10], however, provide evidence that the results of this study are still meaningful. Additionally, limiting the control of propulsion style and collecting more natural, ecologically valid propulsion data better serve the study purpose of examining the kinematics of each propulsion style.

Although the wrist angle calculations, as outlined in this study, are commonly performed, errors in the alignment of the body segment axes are always possible, as noted by Piazza and Cavanagh [24]. This type of misalignment could result in incorrect angles being calculated. Using the Vicon upper limb model, however, safeguards against this error as much as possible by utilizing extra markers on the hand and forearm segments to better align these body segment axes.

This study provides a comparison of the two propulsion conditions in terms of short bouts of linear propulsion. Although analyzing the differences of continuous forward propulsion is an important first step, wheelchair propulsion is often punctuated with frequent stopping, starting, and turning. Additionally, the effects of wheelchair propulsion in contributing to the development of overuse injuries are cumulative. Future studies should include an examination of the effectiveness of the EHDM in improving wrist mechanics during a more complex propulsion protocol completing several different tasks as well as a longitudinal study observing the incidence of CTS in those using a CMW versus the EHDM.

\section{CONCLUSIONS}

The results of this study indicate that EHDM propulsion reduces the occurrence of CTS risk factors compared with CMW use. The EHDM utilizes a more neutral range of wrist orientations than CMW propulsion. Perhaps more importantly, EHDM propulsion consistently utilized an average wrist orientation within the limits of the neutral range of wrist positioning in both planes of motion, as specified by Keir et al. [13]. It is likely that the more neutral range of wrist orientations reduces median nerve compression with EHDM use. Because median nerve damage typical of CTS is cumulative, any reduction in degenerative factors marks an important step toward reducing the likelihood of developing CTS [9].

\section{ACKNOWLEDGMENTS}

\section{Author Contributions:}

Study concept and design: L. A. Zukowski, O. Shechtman, M. D. Tillman. Acquisition of data: L. A. Zukowski, J. A. Roper, D. M. Otzel, P. W. Hovis.

Analysis and interpretation of data: L. A. Zukowski, J. A. Roper, M. D. Tillman.

Drafting of manuscript: L. A. Zukowski, M. D. Tillman. Critical review of manuscript for important intellectual content: J. A. Roper, O. Shechtman, D. M. Otzel, P. W. Hovis.

Statistical analysis: L. A. Zukowski.

Study supervision: M. D. Tillman.

Financial Disclosures: The authors have declared that no competing interests exist. 
Funding/Support: This material was based on work supported by the Clinical and Translational Science Institute Pilot and Collaborative Research Projects, National Institutes of Health (grant UL1 TR000064).

Additional Contributions: Lisa A. Zukowski has received her $\mathrm{PhD}$ and is now affiliated with the Division of Physical Therapy, Department of Allied Health Sciences, University of North Carolina at Chapel Hill, Chapel Hill, North Carolina.

Institutional Review: This study was approved by the University of Florida Institutional Review Board. Participants provided informed consent.

Participant Follow-Up: The authors will contact the study subjects who requested notification of the publication of this article.

\section{REFERENCES}

1. Aljure J, Eltorai I, Bradley WE, Lin JE, Johnson B. Carpal tunnel syndrome in paraplegic patients. Paraplegia. 1985; 23(3):182-86. [PMID:4011294] http://dx.doi.org/10.1038/sc.1985.31

2. Gellman H, Chandler DR, Petrasek J, Sie I, Adkins R, Waters RL. Carpal tunnel syndrome in paraplegic patients. J Bone Joint Surg Am. 1988;70(4):517-19. [PMID:3356717]

3. Sie IH, Waters RL, Adkins RH, Gellman H. Upper extremity pain in the postrehabilitation spinal cord injured patient. Arch Phys Med Rehabil. 1992;73(1):44-48. [PMID:1729973]

4. Pentland WE, Twomey LT. Upper limb function in persons with long term paraplegia and implications for independence: Part I. Paraplegia. 1994;32(4):211-18. [PMID:8022630] http://dx.doi.org/10.1038/sc.1994.40

5. Tanzer RC. The carpal-tunnel syndrome; a clinical and anatomical study. J Bone Joint Surg Am. 1959;41-A(4):626-34. [PMID:13664702]

6. Phalen GS. The carpal-tunnel syndrome. Seventeen years' experience in diagnosis and treatment of six hundred fiftyfour hands. J Bone Joint Surg Am. 1966;48(2):211-28. [PMID:5934271]

7. Lundqvist C, Siösteen A, Blomstrand C, Lind B, Sullivan M. Spinal cord injuries. Clinical, functional, and emotional status. Spine. 1991;16(1):78-83. [PMID:2003241] http://dx.doi.org/10.1097/00007632-199101000-00014

8. Subbarao JV, Klopfstein J, Turpin R. Prevalence and impact of wrist and shoulder pain in patients with spinal cord injury. J Spinal Cord Med. 1995;18(1):9-13. [PMID:7640974]

9. Lundborg G. Nerve injury and repair. Edinburgh (United Kingdom): Churchill Livingstone; 1988.

10. Boninger ML, Cooper RA, Robertson RN, Rudy TE. Wrist biomechanics during two speeds of wheelchair propulsion:
An analysis using a local coordinate system. Arch Phys Med Rehabil. 1997;78(4):364-72. [PMID:9111455] http://dx.doi.org/10.1016/S0003-9993(97)90227-6

11. Hoover AE, Cooper RA, Ding D, Dvorznak M, Cooper R, Fitzgerald SG, Boninger ML. Comparing driving habits of wheelchair users: manual vs. power. Proceedings of the 26th Annual RESNA Conference; 2003 Jun 19-23; Atlanta, GA.

12. Armstrong TJ, Chaffin DB. Some biomechanical aspects of the carpal tunnel. J Biomech. 1979;12(7):567-70. [PMID:479207] http://dx.doi.org/10.1016/0021-9290(79)90045-9

13. Keir PJ, Bach JM, Hudes M, Rempel DM. Guidelines for wrist posture based on carpal tunnel pressure thresholds. Hum Factors. 2007;49(1):88-99. [PMID:17315846] http://dx.doi.org/10.1518/001872007779598127

14. Lundborg G, Gelberman RH, Minteer-Convery M, Lee YF, Hargens AR. Median nerve compression in the carpal tunnel-functional response to experimentally induced controlled pressure. J Hand Surg Am. 1982;7(3):252-59.

[PMID:7086092] http://dx.doi.org/10.1016/S0363-5023(82)80175-5

15. Hargens AR, Romine JS, Sipe JC, Evans KL, Mubarak SJ, Akeson WH. Peripheral nerve-conduction block by high muscle-compartment pressure. J Bone Joint Surg Am. 1979;61(2):192-200. [PMID:217879]

16. Boninger ML, Impink BG, Cooper RA, Koontz AM. Relation between median and ulnar nerve function and wrist kinematics during wheelchair propulsion. Arch Phys Med Rehabil. 2004;85(7):1141-45. [PMID:15241765] http://dx.doi.org/10.1016/j.apmr.2003.11.016

17. Veeger HE, Meershoek LS, van der Woude LH, Langenhoff JM. Wrist motion in handrim wheelchair propulsion. J Rehabil Res Dev. 1998;35(3):305-13. [PMID:9704314]

18. Engel P, Seeliger K. Technological and physiological characteristics of a newly developed hand-lever drive system for wheelchairs. J Rehabil Res Dev. 1986;23(4):37-40. [PMID:3820120]

19. van der Woude LH, Veeger HE, de Boer Y, Rozendal RH. Physiological evaluation of a newly designed lever mechanism for wheelchairs. J Med Eng Technol. 1993;17(6): 232-40. [PMID:8169940] http://dx.doi.org/10.3109/03091909309006331

20. van der Woude LH, Botden E, Vriend I, Veeger D. Mechanical advantage in wheelchair lever propulsion: Effect on physical strain and efficiency. J Rehabil Res Dev. 1997;34(3):286-94. [PMID:9239621]

21. Mukherjee G, Bhowmik P, Samanta A. Effect of chronic use of different propulsion systems in wheelchair design on the aerobic capacity of Indian users. Indian J Med Res. 2005;121(6):747-58. [PMID:16037619] 
22. Tillman MD, Zukowski LA, Otzel DM, Roper JA, Bouwkamp J, Shechtman O. Upper extremity kinematics during ergonomic hand drive wheelchair propulsion. Med Sci Sports Exerc. 2011;43(5S):862.

http://dx.doi.org/10.1249/01.MSS.0000402405.52776.bc

23. Zukowski LA, Roper JA, Shechtman O, Otzel DM, Bouwkamp J, Tillman MD. Comparison of metabolic cost, performance, and efficiency of propulsion using an ergonomic hand drive mechanism and a conventional manual wheelchair. Arch Phys Med Rehabil. 2014;95(3):546-51.

[PMID:24016403]

http://dx.doi.org/10.1016/j.apmr.2013.08.238

24. Piazza SJ, Cavanagh PR. Measurement of the screw-home motion of the knee is sensitive to errors in axis alignment. $\mathrm{J}$ Biomech. 2000;33(8):1029-34. [PMID:10828334] http://dx.doi.org/10.1016/S0021-9290(00)00056-7
Submitted for publication September 23, 2013. Accepted in revised form August 29, 2014.

This article and any supplementary material should be cited as follows:

Zukowski LA, Roper JA, Shechtman O, Otzel DM, Hovis PW, Tillman MD. Wheelchair ergonomic hand drive mechanism use improves wrist mechanics associated with carpal tunnel syndrome. J Rehabil Res Dev. 2014;51(10):1515-24.

http://dx.doi.org/10.1682/JRRD.2013.09.0211

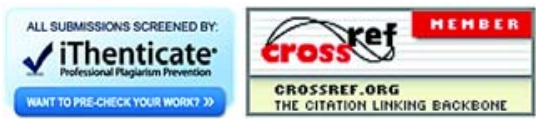


